\title{
Spatiotemporal analysis of tuberculosis incidence and its associated factors in mainland China
}

\author{
C. GUO ${ }^{1,2} \uparrow, \mathrm{Y} \cdot \mathrm{DU}^{1} \uparrow, \mathrm{S} . \mathrm{Q} \cdot \mathrm{SHEN}^{1}, \mathrm{X} . \mathrm{Q} \cdot \mathrm{LAO}^{2}, \mathrm{~J} \cdot \mathrm{QIAN}^{3}$ AND C. Q. OU ${ }^{1 *}$ \\ ${ }^{1}$ State Key Laboratory of Organ Failure Research, Department of Biostatistics, Guangdong Provincial Key \\ Laboratory of Tropical Disease Research, School of Public Health, Southern Medical University, Guangzhou \\ 510515, China \\ ${ }^{2}$ The Jockey Club School of Public Health and Primary Care, Faculty of Medicine, Chinese University of Hong \\ Kong, Hong Kong, China \\ ${ }^{3}$ Department of Mathematics and Physics, School of Biomedical Engineering, Southern Medical University, \\ Guangzhou 510515, China
}

Received 9 January 2017; Final revision 9 May 2017; Accepted 9 May 2017; first published online 9 June 2017

\section{SUMMARY}

Spatiotemporal analysis is an important tool to monitor changes of tuberculosis (TB) epidemiology, identify high-risk regions and guide resource allocation. However, there are limited data on the contributing factors of TB incidence. This study aimed to investigate the spatiotemporal pattern of TB incidence and its associated factors in mainland China during 2005-2013. Global Moran's I test, Getis-Ord Gi index and heat maps were used to examine the spatial clustering and seasonal patterns. Generalized Linear Mixed Model was applied to identify factors associated with TB incidence. TB incidence presented high geographical variations with two main hot spots, while a generally consistent seasonal pattern was observed with a peak in late winter. Furthermore, we found province-level TB incidence increased with the proportion of the elderly but decreased with Gross Demographic Product per capita and the male:female ratio. Meteorological factors also influenced TB incidence. TB showed obvious spatial clustering in mainland China and both the demographic and socio-economic factors and meteorological measures were associated with TB incidence. These results provide the related information to identify the high-risk districts and the evidence for the government to develop corresponding control measures.

Key words: Influencing factors, seasonality, spatiotemporal pattern, tuberculosis.

\section{INTRODUCTION}

Tuberculosis (TB), caused by Mycobacterium tuberculosis, is a devastating infectious disease worldwide

\footnotetext{
* Author for correspondence: State Key Laboratory of Organ Failure Research, Department of Biostatistics, Guangdong Provincial Key Laboratory of Tropical Disease Research, School of Public Health, Southern Medical University, Guangzhou 510515, China.

(Email: ouchunquan@hotmail.com)

$\dagger$ These authors contributed equally.
}

which most commonly affects lungs [1]. TB remains one of the biggest threats due to the low detection and cure rate, drug resistance, and coinfection with other diseases. In 2015, there were $10 \cdot 4$ million new cases and 1.8 million deaths of TB [2]. In China, the morbidity and mortality of TB have remarkably declined under the nationwide practice of a TB control programme (directly observed treatment and short course chemotherapy, DOTS) since 1990 [3]. However, China was still one of the 22 high-burden countries and accounted for $10 \%$ of global TB cases in 2015 [2]. 
Spatiotemporal analysis is an important tool to monitor the changes of TB epidemiology, identify highrisk regions and guide resource allocation. Some studies have shown that the incidence of TB and many other respiratory infectious diseases presents obvious seasonal pattern, which is caused mainly by meteorological factors [4, 5]. Previous studies have focused on temporal analyses of TB using ARIMA (autoregressive integrated moving average) and Fourier series methods [6-8]. However, the temporal patterns vary geographically and simple temporal analysis in one dimension is insufficient to determine this heterogeneity. It is a need to simultaneously analyse the temporal and spatial variations.

Geographical information system (GIS) has been widely applied into epidemiological studies. GIS-based analysis is an efficient and visualised technique to explore the spatial distribution and spatial associations [9-11]. GIS-based methods can efficiently manage geographical data from various sources, including locations, distance and areas [12]. It can provide clear and valuable information for readers to understand and interpret data distribution in a direct way. Another strength of GIS-based methods is the ability of combing geographical data with remote sensing data and traditional statistical methodologies [13]. Recently, two studies in Japan and Portugal reported obvious spatial clustering and seasonal variations of TB based on the Kulldorff's scan statistics $[14,15]$. The Moran's $I$ test and Getis-Ord index are widely used to display the spatial distribution of other infectious diseases [16, 17]. However, all these methods are just used to show the spatial patterns and seasonal variations. There is limited information on the potential influencing factors like spatiotemporal patterns. Some studies performed cross-sectional analysis using linear, Poisson and logistic regression models [18-20], which cannot control for the autocorrelation within cities. Cao et al. [21] applied the Bayesian framework to estimate the effect of province-level annual meteorological measures on TB in mainland China. However, results of Bayesian analysis are dependant on priors, which may be chosen based on personal experience. Generalized Linear Mixed Model (GLMM) is an alternative way to estimate parameters in multiple levels (e.g. within- and between-province level). Particularly, the role of demographic and socioeconomic factors in spatial heterogeneity of TB incidence need to be further studied and meteorological effects may be confounded without adjusting for these factors in the model. Such important province-level demographical and socio-economic factor were not analysed and controlled for in Cao's study, which may lead to a bias in estimating meteorological effects. However, Cao et al. [21] observed that TB incidence was negatively associated with annual wind but positively associated air pressure, but they did not consider demographic and socio-economic factors which may be associated with spatial heterogeneity of TB incidence and confound the meteorological effects.

In China, there were some studies conducted in a single city or small territories [22, 23], in which environmental and socio-economic gradients are limited to detect the related factors of spatial patterns of TB. Recently, Cao et al. [21] found the spatial patterns of TB in mainland China were linked to meteorological factors. Amongst potential contributing factors of TB, socio-economic factors are modifiable but underexplored in China, strengthening the importance of identifying socio-economic factors of TB and quantifying the effects in China.

This study aimed to investigate the spatial clustering patterns and seasonal variations of TB across different provinces and study years. This study also intended to analyse the demographic, socio-economic as well as meteorological factors associated with TB incidence in the whole mainland China during 2005-2013.

\section{METHODS}

\section{Study sites}

The present study included a total of 31 provinces in mainland China (excluding Taiwan, Hong Kong and Macao). China is the largest country in population and ranks the third in area in the world. There are temperate, subtropical and tropical climates and various economic situations in the 31 provinces.

\section{Data source}

In mainland China, the TB Information Management System has been established and operated by CCDC (Chinese Centre for Disease Control and Prevention) since 2004. It is mandatory to report every single TB case through this on-line system. The monthly reported all-forms TB cases from January 2005 to December 2013 in each of 31 provinces of mainland China were obtained from the Data-centre of China Public Health Science (http://www.phsciencedata.cn/). This study included all reported TB cases including HIV-positive TB patients. The data in 2004 were excluded due to the large number of missing data in 
some provinces at the beginning of the implementation of the monitoring system. National Bureau of Statistics of China provided yearly demographic and socio-economic data for each provinces during 20052013, including population (100 000 persons), Gross Demographic Product (GDP) per capita (10000 $\mathrm{RMB})$, the proportion of the elderly (\%), the ratio of male to female (M:F ratio, $1 \%$ ), number of doctors per 1000 persons, number of inpatient beds per 1000 persons, the proportion of people with high education (college or above, \%), and population density (persons per square kilometres).

Monthly meteorological data for each province during the study period were obtained from the China Meteorological Data Sharing Service System (http:// data.cma.cn/). Average meteorological measures can provide more easily interpreted results in a policy context and are more familiar to the public. Therefore, they are widely used as indicators of meteorological factors in studies of meteorological effects on infectious diseases [5, 24]. The monthly average temperature $\left({ }^{\circ} \mathrm{C}\right)$, duration of sunshine (hours), precipitation $(\mathrm{mm})$, average wind speed $(\mathrm{m} / \mathrm{s})$, average atmospheric pressure $(\mathrm{hPa})$ and average relative humidity $(\%)$ were included in this study.

\section{Statistical analysis}

We used GIS to present the spatial pattern of annual TB incidence in 31 provinces during 2005-2013. The Global Moran's $I$ test was used to determine whether there is any spatial autocorrelation nationally. The Global Moran's $I$ test has been employed frequently to investigate spatial clustering of infectious diseases $[25,26]$.

The Global Moran's $I$ index is

$I=\frac{n}{S_{0}} \frac{\sum_{i=1}^{n} \sum_{j=1}^{n} w_{i, j} Z_{i} Z_{j}}{\sum_{i=1}^{n} Z_{i}^{2}}$

where $Z_{i}=\left(x_{i}-\bar{X}\right), w_{i, j}$ is the spatial weight between feature $i$ and $j$, and $n$ is the total number of features.

$S_{0}=\sum_{i=1}^{n} \sum_{j=1}^{n} w_{i, j}$

$Z$ statistic is the standardisation of Moran's $I$ :

$Z=\frac{I-E(I)}{\sqrt{V(I)}}$.

Previous literature pointed out that it is less sensitive to the spatial weight matrix and thus more popular than Kulldorff's spatial scan statistic [27].
Getis-Ord Gi index was performed to detect the hot spot of TB incidence at province level, by testing whether there is any significant correlation between a province and its neighbouring provinces [27].

To examine the seasonal pattern of TB incidence, the heat maps were used to show monthly variations of TB incidence by province and by year [5]. This approach could quantify and visualise the seasonality after adjusting for the sampling differences across provinces and over years by dividing yearly cases for each province and averaging cases of all the provinces for each year.

Spearman rank correlation was utilised to analyse the correlation between potential explanatory factors of TB incidence. We conducted the GLMM to examine the effects of meteorological, demographic, and socio-economic factors on TB incidence [28]. The monthly number of TB cases was assumed to follow the Poisson distribution. Therefore, we constructed a Poisson regression model with the log population term as an offset to control for population variations across provinces and over study duration. Considering the correlations of TB occurrences among people within the same province and large differences in the magnitudes of economic level and meteorological environment among provinces, we treated province as a random effect. Season (spring: March-May, summer: June-August, autumn: September-November, and winter: December-February) as an indicator variable and linear and quadratic terms of year were considered to control for the seasonality and trend of TB occurrence, respectively. The residual spatial autocorrelation was controlled by including the latitude and longitude [29]. All the explanatory factors were determined in the final model by a stepwise backward deletion test [30]. The final model can be specified as follows:

$$
\begin{aligned}
\log \left(E\left(Y_{i t}\right)\right)= & \operatorname{offset}\left(\log \left(\operatorname{pop}_{i j}\right)\right)+a_{i} \\
& +\operatorname{sex}_{i j}+\operatorname{old}_{i j}+\operatorname{gdp}_{i j}+\operatorname{temp}_{i t} \\
& +\operatorname{wind}_{i t}+\operatorname{press}_{i t}+\text { humidit }_{i t}+\text { season } \\
& + \text { year }+ \text { year }^{2}+\text { longitude }_{i}
\end{aligned}
$$

where $\operatorname{pop}_{i j}$ is the population of province $i(i=1,2, \ldots$, $31)$ in year $j(j=2005,2006, \ldots, 2013)$ and $a_{i}$ is the random intercept for the province $i . Y_{i t}$ is the number of TB cases in province $i$ in month $t(t=1,2, \ldots, 96)$. The $\operatorname{sex}_{i j}, \operatorname{old}_{i j}$ and $\operatorname{gdp}_{i j}$ indicate yearly M:F ratio, the proportion of the elderly, and GDP per capita in province $i$ in year $j$, respectively. The temp $i t$, wind $_{i t}$, ,ress $_{i t}$ 
and humid ${ }_{i t}$ represent monthly mean temperature, wind speed, atmospheric pressure and relative humidity in month $t$ in province $i$, respectively. The excess risk (ER) percentage of $\mathrm{TB}$ incidence with the 95\% confidence interval were calculated for all explanatory variables. We did the sensitivity analyses by adding a quadratic term of longitude and excluding the quadratic term of year to check whether there was non-linear association between longitude and TB occurrence and whether a linear trend was enough to control for the long-term trend in TB incidence in study period (Supplementary Table S1). The Global Moran's I test, Getis-Ord Gi index analysis and visualised maps of TB incidence were made based on ArcGIS software version 10.1. The heat map and the Poisson mixed model based on 'lme4' package were conducted in $\mathrm{R}$ language version 3.3.2.

\section{RESULTS}

A total of 9597884 TB cases were reported in mainland China from January 2005 to December 2013, with an average annual incidence of $80 \cdot 3$ cases per 100000 persons and an annual mortality rate of 23.50 deaths per 10000000 persons (Fig. 1). A gradually decreasing trend in incidence and mortality was observed during the study period of 2005-2013. The average annual incidence of TB for the 31 provinces is presented in Fig. 2. There were obvious spatial variations of $\mathrm{TB}$ incidence in mainland China, with the highest incidence in Xinjiang, Guizhou and Hainan.

There were consistently high spatial autocorrelations of TB incidence with the Global Moran's

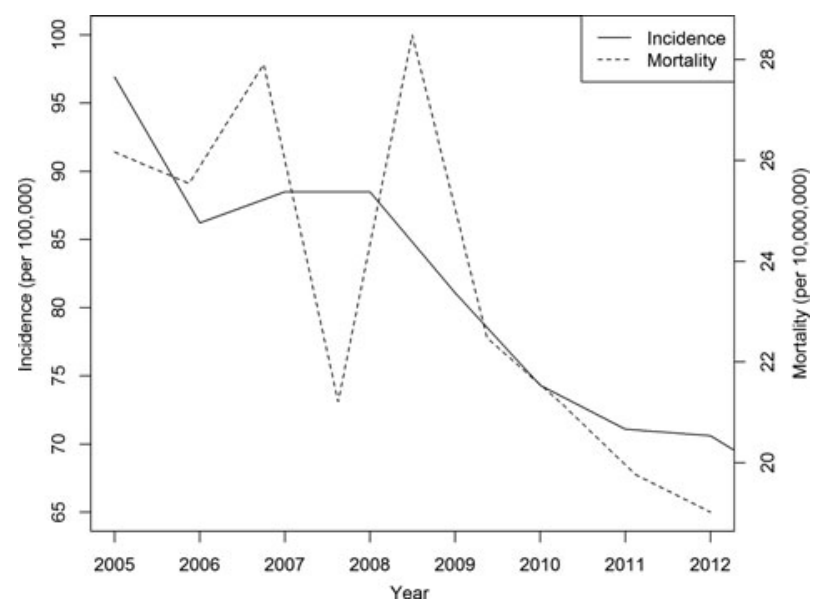

Fig. 1. The annual incidence rate and mortality of TB by year in mainland China in 2005-2013.
$I$ index ranging from 0.255 to $0.410(P<0.001)$ in each year from 2005 to 2013 . The average annual incidence of TB during the study period also had a significant spatial autocorrelation $(Z=5 \cdot 761, P<0 \cdot 001)$ (Table 1).

The hot spot analysis illustrated two main hot spots (provinces with high TB incidence clustered) and one cold spot (provinces with low TB incidence clustered) in the study duration in mainland China (Fig. 3). The hot spots dominantly clustered in Northwest (Xinjiang, Qinghai, Tibet, and Yunnan) and central China (Hunan). That is, these provinces had significantly higher TB incidence than other surrounding provinces. The hot spots gradually transferred from central China to the Northwest during the study period. None of the hot spots in central China were statistically significant at the 0.05 level since 2010 . The cold spots stably clustered in the provinces located in East China, including Beijing, Tianjin, Hebei and Jiangsu.

Heat maps show the median rate of TB incidence in each month of the year across 31 provinces. It revealed generally consistent seasonal patterns of $\mathrm{TB}$ across mainland China (Fig. 4a). In northern and southern provinces, TB cases showed an annual peak in late winter to spring (January-May) and a trough in early winter (December), except for a moderately low point found in February (Fig. 4a). The empirical peaks were in late winter for Northern and Southern provinces. Figure $4 b$ showed the heat map of monthly median incidence of TB in the whole study area by year. A similar seasonality pattern was observed in each year from 2005 to 2008, but the seasonality was relatively ambiguous and the peak amplitudes decreased dramatically after 2009 (Fig. 4b).

The descriptive statistics of potential influencing factors for each province are shown in Supplementary Table S2. The results of bivariate Spearman correlation analyses (Supplementary Table S3) showed there were high correlations between GDP, doctors numbers, beds numbers and high education; population density and mean atmospheric pressure; temperature, water vapour and precipitation; relative humid and sun hour; relative humidity, precipitation and water vapour; (Spearman $\rho>0 \cdot 6, P<0 \cdot 001$ ). Highly correlated variables were not simultaneously included in the model to avoid collinearity and subsequent biases in parameters estimates.

The GLMM revealed significant effects of demographic, socio-economic and meteorological factors on TB incidence (Table 2). The TB incidence decreased with the $\mathrm{M}: \mathrm{F}$ ratio while increased with the proportion 


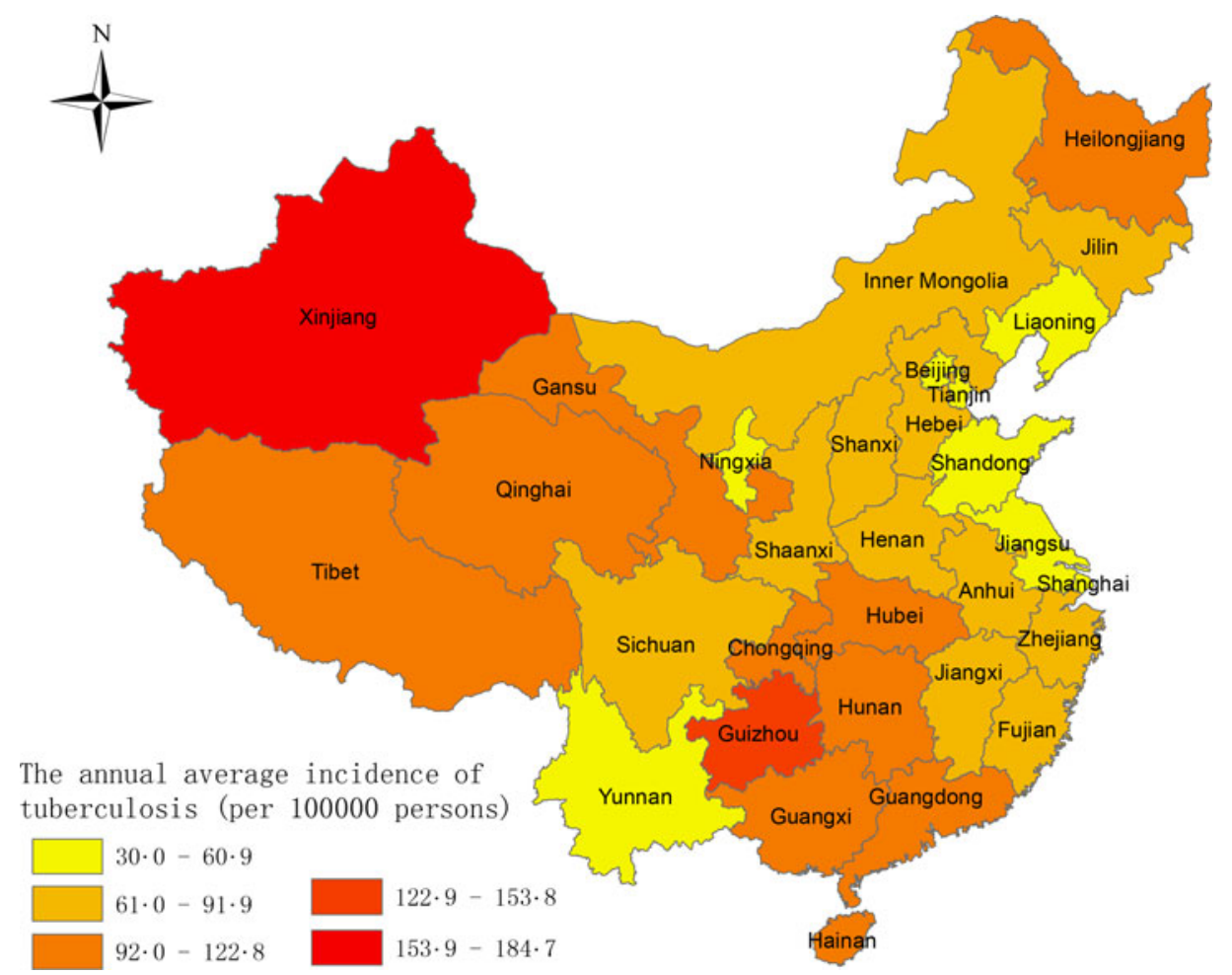

Fig. 2. The average annual incidence of TB by province in mainland China in 2005-2013.

of the elderly. TB incidence decreased $1 \cdot 75 \%(\mathrm{ER} \%=-$ $1 \cdot 75 \%, 95 \%$ CI $-1 \cdot 96 \%$ to $-1 \cdot 54 \%$ ) associated with each 10000 RMB increase in GDP per capita. Besides, mean temperature, wind speed and atmospheric pressure had consistently negative effects on TB incidences, while relative humidity was positively associated with TB. There was a higher incidence risk of $\mathrm{TB}$ in spring $(\mathrm{ER} \%=24 \cdot 80 \%, 95 \%$ CI $24 \cdot 48$ $25 \cdot 12 \%)$ and summer $(\mathrm{ER} \%=8 \cdot 06 \%, 95 \%$ CI $7 \cdot 65-$ $8.48 \%$ ) compared with that in winter. And autumn had a $6 \cdot 05 \%$ decrease $($ ER $\%=-6 \cdot 05 \%, 95 \%$ CI $-6 \cdot 30 \%$ to $5 \cdot 79 \%$ ) in TB incidence than winter. A nonlinear trend in TB incidence was found in China during 2005-2013. Additionally, TB incidence was higher in provinces at a higher longitude $(\mathrm{ER} \%=4 \cdot 56 \%, 95 \%$ CI $1 \cdot 67-7 \cdot 53 \%$ ).

\section{DISCUSSION}

TB is still a major infectious disease in mainland China with around one million cases per year. We found significant spatial clusters of TB incidence in mainland China. The number of hot spots increased during the study period, which was in line with the previous study in Beijing, China [22]. This is reasonable that neighbouring provinces may have effects on each other through the population morbidity, and through sharing similar dietary, living habits and environmental conditions. On the other hand, a remarkable geographic inequality in TB incidence was observed. The economic polarisation promoted the clustering of cities in terms of TB incidence, and the differentials seem to become more obvious. The areas with a high TB incidence clustered in Northwest China, possibly due to underdeveloped economic conditions, poor health care, and ignorance of necessary prevention knowledge [31]. Fortunately, the epidemic has been controlled well in central China since 2010 due to a series of TB control policies and measures implemented by the local authorities, including TB-related health education for residents, periodical training for medical staff, the newly established clinics, free treatment for TB patients, and the improved monitoring or supervision system. Cold spots clustered around the Yangtze River delta, where developed economy, advanced health care, and the widespread awareness of TB-related knowledge were in favour of TB control. Intense surveillance and sufficient health resources should be allocated to the hot spots provinces to narrow down health inequality nationally and reduce the flow-out infected cases to neighbouring cities.

TB incidence showed consistent seasonality in 31 provinces in mainland China with a peak in late winter to spring. Similar seasonality was also observed in 
Table 1. Global Moran's I index for TB incidence in mainland China in 2005-2013

\begin{tabular}{llll}
\hline \hline Year & Moran's $I$ index* & $Z$ statistic & $P$ value \\
\hline 2005 & $0 \cdot 387$ & $5 \cdot 481$ & $<0 \cdot 001$ \\
2006 & $0 \cdot 367$ & $5 \cdot 288$ & $<0 \cdot 001$ \\
2007 & $0 \cdot 385$ & $5 \cdot 523$ & $<0 \cdot 001$ \\
2008 & $0 \cdot 328$ & $4 \cdot 785$ & $<0 \cdot 001$ \\
2009 & $0 \cdot 367$ & $5 \cdot 326$ & $<0 \cdot 001$ \\
2010 & $0 \cdot 399$ & $5 \cdot 704$ & $<0 \cdot 001$ \\
2011 & $0 \cdot 410$ & $5 \cdot 815$ & $<0 \cdot 001$ \\
2012 & $0 \cdot 365$ & $5 \cdot 299$ & $<0 \cdot 001$ \\
2013 & $0 \cdot 255$ & $3 \cdot 827$ & $<0 \cdot 001$ \\
Annual average & $20 \cdot 391$ & $5 \cdot 634$ & $<0 \cdot 001$ \\
\hline \hline
\end{tabular}

* The Global Moran's I index represents the spatial autocorrelation of TB incidences among provinces.

several other countries, such as USA and Mongolia [4, 32]. This peak is mainly driven by meteorological factors and the weather-associated human behaviours. In cold seasons, having less outdoor activities may lead to indoor crowdedness and air pollution, and the deficiency of vitamin D intake through sun exposure
[33, 34], contributing to the TB transmission and increasing the infection risk. In winter, the relatively weak immune system may lead to higher reactivation rate of the latent TB. A latent period of approximately 3-8 weeks for TB infection and the delay in diagnosis and notification may explain that the peak started in late winter rather than at the beginning of cold season. However, Douglas et al. [33] and Leung et al. [23] observed a major peak in summer in UK and Hong Kong. This may be related to the seasonal immigrants in these countries, but the potential mechanism needs to be explored further. This seasonality of TB incidence was closely associated with meteorological factors. Temperature had negative effects on TB incidence, supported by other research in Nigeria and North India [7, 35]. High temperature may restrain the development of TB via affecting people's blood pressure, aspiration and biological vitality to increase the infection rate [36]. Also, the recombinant strain of Mycobacterium can stop growing and even could be destroyed when temperature exceeded $37^{\circ} \mathrm{C}$ [37]. Consistent with previous research [38, 39], we found $\mathrm{TB}$ incidence increased with relative
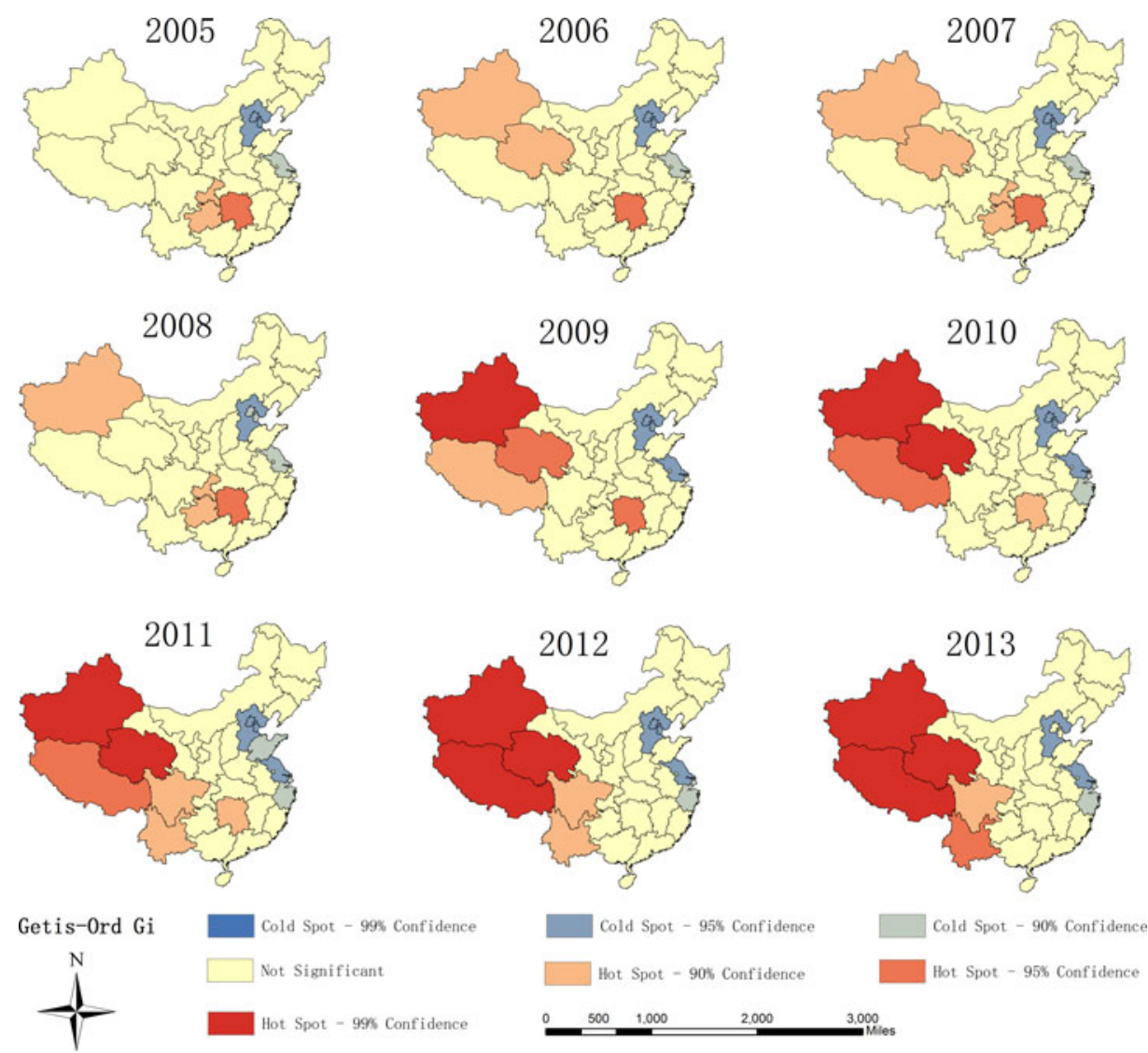

Fig. 3. The hot spot analysis of TB incidence in mainland China in 2005-2013. 

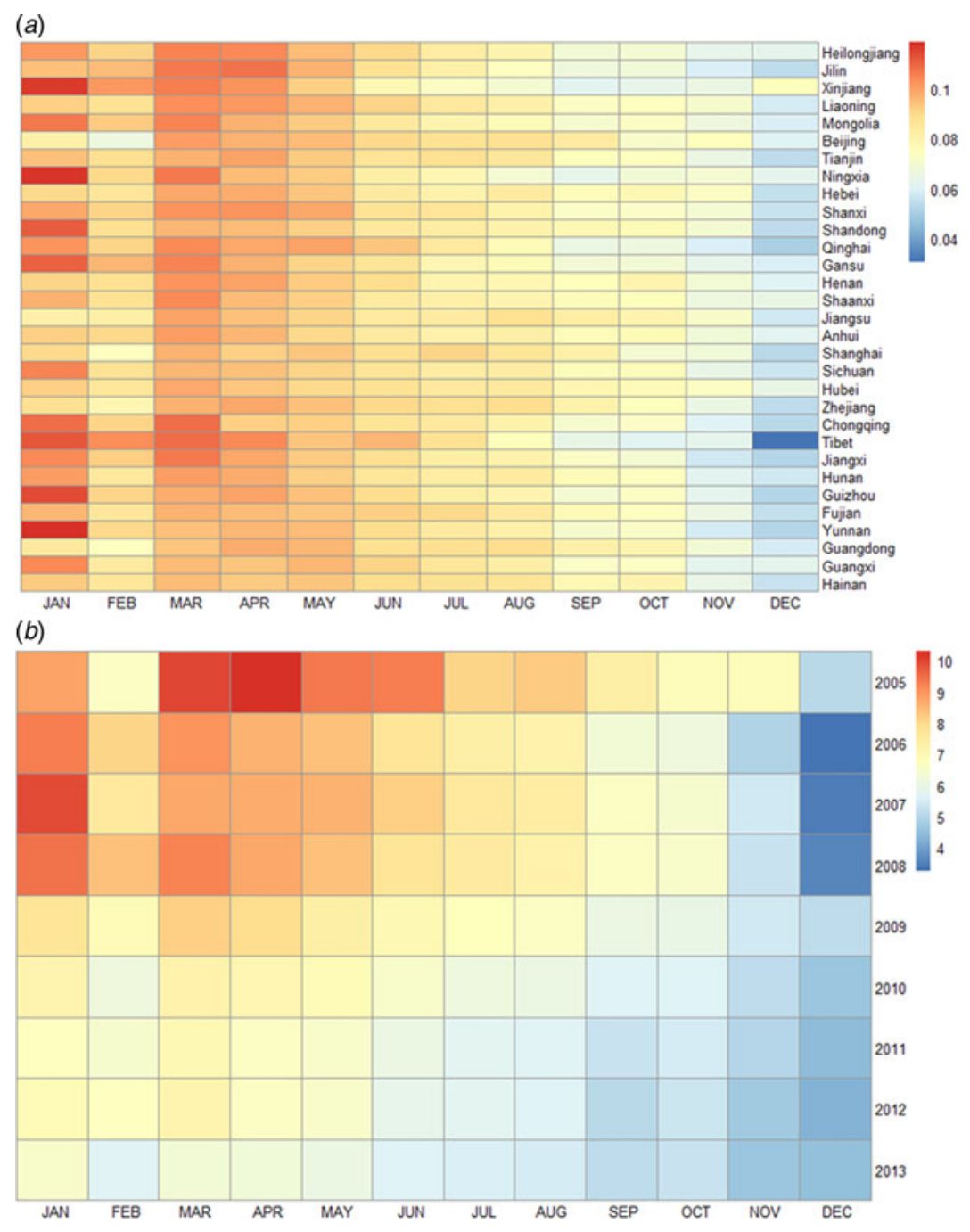

Fig. 4. Heat maps of TB incidence by province and by year in 2005-2013. (a) Seasonality of TB incidence standardised by the annual TB incidence for 31 provinces listed by their latitudes from the top to the bottom; and $(b)$ the seasonality of TB incidence for each year in mainland China.

humidity. High relative humidity may provide a favourable environment for Mycobacterium to be attached to ambient particulates and stay in the air for a long time. Additionally, high relative humidity can exert profound effects on immunity-oriented problems through restricting the metabolism rate. Few studies examined the association between wind speed, atmospheric pressure and TB. In this study, we found wind and atmospheric pressure were protective factors of TB, consistent with the findings reported by Cao et al. [21]. Low speed of wind may contribute to the spread of Mycobacterium floating in the wind, but the high wind speed could decrease TB incidence by releasing people's susceptibility to extreme weathers and accelerate the air ventilation. Danet et al. [40] found an unhealthy effect of high atmospheric pressure on the occurrence of coronary events. However, Cao et al. [21] observed that TB incidence was negatively associated with annual wind but positively associated with air pressure, but they did not consider demographic and socio-economic factors which may confound the estimates of meteorological effects since these factors were found to be associated with TB incidence in our study.

We observed the M:F ratio was negatively associated with TB incidence. An early study in 1952 in England and Wales also reported an increased TB incidence with the proportion of females [41]. However, Neyrolles and Quintana-Murci [42] pointed out a higher TB incidence in males, mainly because of higher rate of tobacco use. These differences in males and females had a close relation with sex steroids and the 
Table 2. Percentage change (\%) in risk of TB incidence in mainland China, 2005-2013

\begin{tabular}{|c|c|c|c|}
\hline Variables & Excess risk (\%) & $Z$ statistic & $P$ value \\
\hline$M: F$ ratio $(1 \%)$ & $-0 \cdot 35(-0 \cdot 39,-0 \cdot 32)$ & $-20 \cdot 32$ & $<0.001$ \\
\hline Proportion of the elderly (\%) & $0.86(0.73,0.99)$ & $12 \cdot 90$ & $<0.001$ \\
\hline GDP per capita (10 $000 \mathrm{RMB})$ & $-1.75(-1.96,-1.54)$ & $-16 \cdot 32$ & $<0.001$ \\
\hline Temperature $\left({ }^{\circ} \mathrm{C}\right)$ & $-0.77(-0.79,-0.75)$ & $-79 \cdot 41$ & $<0.001$ \\
\hline Wind speed $(\mathrm{m} / \mathrm{s})$ & $-1.94(-2 \cdot 11,-1 \cdot 77)$ & $-21 \cdot 57$ & $<0.001$ \\
\hline Atmospheric pressure $(\mathrm{hPa})$ & $-0.92(-0.94,-0 \cdot 90)$ & $-75 \cdot 01$ & $<0 \cdot 001$ \\
\hline Relative humidity (\%) & $0.06(0.05,0.07)$ & $15 \cdot 09$ & $<0.001$ \\
\hline Winter (reference) & - & - & - \\
\hline Spring & $24 \cdot 80(24 \cdot 48,25 \cdot 12)$ & $169 \cdot 99$ & $<0.001$ \\
\hline Summer & $8 \cdot 06(7 \cdot 65,8 \cdot 48)$ & $39 \cdot 64$ & $<0 \cdot 001$ \\
\hline Autumn & $-6 \cdot 05(-6 \cdot 30,-5 \cdot 79)$ & $-45 \cdot 42$ & $<0.001$ \\
\hline Year & $-3 \cdot 91(-3 \cdot 99,-3 \cdot 83)$ & -91.96 & $<0 \cdot 001$ \\
\hline Year $\times$ year & $-0.07(-0.08,-0.06)$ & $-12 \cdot 07$ & $<0.001$ \\
\hline Longitude $\left({ }^{\circ}\right)$ & $4 \cdot 56(1 \cdot 67,7 \cdot 53)$ & $3 \cdot 46$ & $<0 \cdot 001$ \\
\hline
\end{tabular}

antimycobacterial immune responses [42]. Onozuka and Hagihara [43] and Randremanana et al. [11] did not find sex differences in the TB incidence in Japan and Antananarivo, Madagascar, respectively. Khaliq et al. [20] illustrated young females (under 24 years old) had higher TB notification than young males, but there was an opposite situation for older age groups.

We found TB incidence increased with the proportion of the elderly, which is supported by previous studies [35, 44]. For the elderly, the poor physical situation with other chronic diseases and weak immune system make them vulnerable to be infected with TB. For example, previous studies reported an extremely higher incidence of AIDS (acquired immunodeficiency syndrome) in the elderly than in young people, associated with a higher TB incidence [45, 46]. Also, MacLaughlin and Holick [47] found that the elderly produced less vitamin D in their skins, which was an important factor to prevent the TB. Additionally, the M. tuberculosis could be reactivated with increasing age under appropriate condition. A molecular biology study reported this may occur even after 33 years since infection [48]. Therefore, the likelihood of TB infection and the incidence of active TB increase definitely with age.

In this study, we found a negative association between province-level GDP per capita and TB incidence, indicating that the local economic development is beneficial to the control of TB. Similar results were observed for other infectious diseases [49, 50]. People live in impoverished cities may have less access to TB-related knowledge and health care services, which leads to a higher risk of $\mathrm{TB}$ infection.
Reducing poverty is essential for ending the global TB epidemic and the elimination of TB.

This study has several limitations. First, although the official surveillance system of TB has been established since 2004 in China and each case is required to be reported, the potential under-reported cases are inevitable. Meanwhile, among all TB cases reported, only $40 \cdot 26 \%$ were bacteriologically confirmed and the others were clinically diagnosed cases. Some infections such as non-tuberculous mycobacteria which have some similar clinical symptoms with TB may be misdiagnosed as TB. Second, in this ecological study, we found several socio-demographic and economic factors associated with TB. However, the longitude variable was statistically significant in the model, indicating that the observed variables cannot explain adequately the spatial differences in TB in mainland China. There are some other risk factors, such as individual, social, clinical and behavioural factors associated with TB incidence that were not considered due to the unavailability of data. Third, the inherent limitation in GIS-based methods may cause the uncertainty of results, such as different weights, study unit and estimate methods. In the present study, we used GIS-based method just to visually explore the spatial patterns of TB and further constructed a GLMM to determine factors associated with the spatiotemporal variations. Finally, this national analysis utilised the monthly TB data of 31 provinces in mainland China, but further studies with higher resolution in spatial and temporal dimensions, such as daily data at the city level, would provide more comprehensive and precise information of $\mathrm{TB}$ and socio-economic gradients to 
better understand the spatiotemporal patterns of TB incidence and the influencing factors.

In conclusion, TB incidence generally peaked in late winter and spatially clustered with two hot spots and one cold spot in mainland China. Both demographic and socio-economic factors and meteorological measures were associated with TB incidence. This study would help gain a better understanding of the spatiotemporal patterns of TB and provide valuable information for government or related health organisations to initiate corresponding measures before seasonal peaks of TB incidence and to identify priority TB control objects for the population and regions with high incidence risk such as the elderly and those with low socioeconomic conditions.

\section{SUPPLEMENTARY MATERIAL}

The supplementary material for this article can be found at https://doi.org/10.1017/S0950268817001133

\section{ACKNOWLEDGEMENTS}

This work was supported by National Nature Science Foundation of China [Grant no. 81573249], Nature Science Foundation of Guangdong Province [Grant no. 2016A030313530], and Science and Technology Planning Project of Guangdong Province, China [Grant numbers 2013B021800041 and 2013B060500046].

\section{DECLARATION OF INTEREST}

None.

\section{ETHICS APPROVAL AND CONSENT TO PARTICIPATE}

The authors assert that all procedures contributing to this work comply with the Ethics Committee of Southern Medical University, where this study was conducted. Informed consent was not required because the data of monthly number of TB cases were obtained from official website and all data were anonymous.

\section{REFERENCES}

1. Sharma A, et al. Tuberculosis caused by Mycobacterium africanum, United States, 2004-2013. Emerging Infectious Diseases 2016; 22: 396-403.

2. World Health Report. Global Tuberculosis Report 2015. Geneva: World Health Organization, 2016.
3. Squire SB, Tang S. How much of China's success in tuberculosis control is really due to DOTS? The Lancet 2004; 364: 391-392.

4. Willis MD, et al. Seasonality of tuberculosis in the United States, 1993-2008. Clinical Infectious Diseases 2012; 54: 1553-1560.

5. Yu H, et al. Characterization of regional influenza seasonality patterns in China and implications for vaccination strategies: spatio-temporal modeling of surveillance data. PLoS Medicine 2013; 10: e1001552.

6. Atun RA, et al. Seasonal variation and hospital utilization for tuberculosis in Russia: hospitals as social care institutions. European Journal of Public Health 2005; 15: $350-354$.

7. Narula P, et al. Analyzing seasonality of tuberculosis across Indian states and union territories. Journal of Epidemiology Global Health 2015; 5: 337-346.

8. Yi J, et al. Applications of multiple seasonal autoregressive integrated moving average (ARIMA) model on predictive incidence of tuberculosis. Zhonghua Yu Fang Yi Xue Za Zhi, 2007; 41: 118-121.

9. Ray N, Lehmann A, Joly P. Modeling spatial distribution of amphibian populations: a GIS approach based on habitat matrix permeability. Biodiversity and Conservation 2002; 11: 2143-2165.

10. Getis A, Ord JK. The analysis of spatial association by use of distance statistics. Geographical Analysis 1992; 24: 189-206.

11. Randremanana RV, et al. Spatial clustering of pulmonary tuberculosis and impact of the care factors in Antananarivo City. Tropical Medicine \& International Health 2009; 14: 429-437.

12. Moore DA, Carpenter TE. Spatial analytical methods and geographic information systems: use in health research and epidemiology. Epidemiologic Reviews 1999; 21: 143-161.

13. Bocco G, Mendoza M, Velázquez A. Remote sensing and GIS-based regional geomorphological mapping a tool for land use planning in developing countries. Geomorphology 2001; 39: 211-219.

14. Onozuka D, Hagihara A. Geographic prediction of tuberculosis clusters in Fukuoka, Japan, using the space-time scan statistic. BMC Infectious Disease 2007; 7: 26.

15. Nunes C. Tuberculosis incidence in Portugal: spatiotemporal clustering. International Journal of Health Geographics 2007; 6: 30.

16. Wang H, et al. Detecting the association between meteorological factors and hand, foot, and mouth disease using spatial panel data models. International Journal of Infectious Diseases 2015; 34: 66-70.

17. Tran A, et al. Dengue spatial and temporal patterns, French Guiana, 2001. Emerging Infectious Diseases 2004; 10: 7 .

18. Pealing $\mathbf{L}$, et al. Risk of tuberculosis in patients with diabetes: population based cohort study using the UK Clinical Practice Research Datalink. BMC Medicine 2015; 13: 135.

19. Li XX, et al. Comparing risk factors for primary multidrug-resistant tuberculosis and primary drugsusceptible tuberculosis in Jiangsu province, China: a 
matched-pairs case-control study. American Journal of Tropical Medicine and Hygiene 2015; 92: 280-285.

20. Khaliq A, Batool SA, Chaudhry MN. Seasonality and trend analysis of tuberculosis in Lahore, Pakistan from 2006 to 2013. Journal of Epidemiology Global Health 2015; 5: 397-403.

21. Cao K, et al. Spatial-temporal epidemiology of tuberculosis in Mainland China: an analysis based on Bayesian theory. International Journal of Environmental Research and Public Health 2016; 13: 469.

22. Liu Y, et al. Investigation of space-time clusters and geospatial hot spots for the occurrence of tuberculosis in Beijing. International Journal of Tuberculosis Lung Disease 2012; 16: 486-491.

23. Leung CC, et al. Seasonal pattern of tuberculosis in Hong Kong. International Journal of Epidemiology 2005; 34: 924-930.

24. Guo C, et al. Malaria incidence from 2005-2013 and its associations with meteorological factors in Guangdong, China. Malaria Journal 2015; 14: 116.

25. Fosgate GT, et al. Time-space clustering of human brucellosis, California, 1973-1992. Emerging Infectious Diseases 2002; 8: 672-678.

26. Martins-Melo FR, et al. Spatiotemporal patterns of schistosomiasis-related deaths, Brazil, 2000-2011. Emerging Infectious Diseases 2015; 21: 1820.

27. Getis A. Spatial interaction and spatial autocorrelation: a cross-product approach. Environment and Planning $A$ 1991; 23: 1269-1277.

28. Brown H, Prescott R. Applied Mixed Models in Medicine. England: John Wiley \& Sons, 2014.

29. Uejio CK, et al. Climatic influences on Cryptococcus gattii populations, Vancouver Island, Canada, 2002-2004. Emerging Infectious Diseases 2015; 21: 1989-1996.

30. Galanis E, et al. Epidemiology of Cryptococcus gattii, British Columbia, Canada, 1999-2007. Emerging Infectious Diseases 2010; 16: 251-257.

31. Delahay R, et al. The spatio-temporal distribution of Mycobacterium bovis (bovine tuberculosis) infection in a high-density badger population. Journal of Animal Ecology 2000; 69: 428-441.

32. Naranbat $\mathbf{N}$, et al. Seasonality of tuberculosis in an Eastern-Asian country with an extreme continental climate. European Respiratory Journal 2009; 34: 921-925.

33. Douglas AS, Strachan DP, Maxwell JD. Seasonality of tuberculosis: the reverse of other respiratory diseases in the UK. Thorax 1996; 51: 3.

34. Cohen A, Mehta S. Pollution and tuberculosis: outdoor sources. PLoS Medicine 2007. Published online: 27 Mar 2007. doi: 10.1371/ journal. pmed. 0040142 .

35. Omonijo AG, et al. A study of weather related respiratory diseases in Eco-climatic zones. African Physical Review 2001; 5: 16.
36. Petersen WF. Tuberculosis: weather and resistance, In Annual Meeting of the American College of Chest Physicians, Atlantic City, New Jersey, 1942.

37. Pinto CT, Nano FE. Stable, temperature-sensitive recombinant strain of Mycobacterium smegmatis generated through the substitution of a psychrophilic ligA gene. FEMS Microbiology Letters 2015; 362: e1-e6.

38. Blanco J, Kheradmand H. Climate variability and population health in China: updated knowledge, challenges and opportunities. In Climate Change-Socioeconomic Effects. Rijeka, Croatia: InTech, 2011, pp. 81-102.

39. Nardell EA. Catching droplet nuclei: toward a better understanding of tuberculosis transmission. American Journal of Respiratory and Critical Care Medicine 2004; 169: 553-554.

40. Danet S, et al. Unhealthy effects of atmospheric temperature and pressure on the occurrence of myocardial infarction and coronary deaths. Circulation 1999. Published online 6 Jul 1999; 100: e1-e7

41. Styblo K. Tuberculosis surveillance research unit of the IUAT. In Progress Report, 1973.

42. Neyrolles O, Quintana-Murci L. Sexual inequality in tuberculosis. PLoS Medicine 2009. Published online 22 Dec 2009. doi: 10.1371/ journal. pmed. 1000199.

43. Onozuka D, Hagihara A. The association of extreme temperatures and the incidence of tuberculosis in Japan. International Journal of Biometeorology 2015; 59: 1107-1114.

44. Tuite AR, et al. Epidemiological evaluation of spatiotemporal and genotypic clustering of Mycobacterium tuberculosis in Ontario, Canada. International Journal of Tuberculosis and Lung Diseases 2013; 17: 1322-1327.

45. Chaimowicz F. Age transition of tuberculosis incidence and mortality in Brazil. Revista de Saúde Pública 2001; 35: 81-87.

46. Sonnenberg $\mathbf{P}$, et al. How soon after infection with HIV does the risk of tuberculosis start to increase? A retrospective cohort study in South African gold miners. Journal of Infectious Diseases 2005; 191: 150-158.

47. MacLaughlin J, Holick MF. Aging decreases the capacity of human skin to produce vitamin D3. Journal of Clinical Investigation 1985; 76: 1536.

48. Lillebaek T, et al. Molecular evidence of endogenous reactivation of Mycobacterium tuberculosis after 33 years of latent infection. Journal of Infectious Diseases 2002; 185: 401-404.

49. Xu W, et al. Adherence to anti-tuberculosis treatment among pulmonary tuberculosis patients: a qualitative and quantitative study. BMC Health Services Research 2009; 9: 169.

50. Brownstein JS, et al. Information technology and global surveillance of cases of $2009 \mathrm{H} 1 \mathrm{~N} 1$ influenza. New England Journal of Medicine 2010; 362: 1731-1735. 